\title{
A rational approximation to the evolution of a free surface during fluid withdrawal through a point sink
}
G. C. Hocking ${ }^{1}$
T. E. Stokes ${ }^{2}$
L. K. Forbes ${ }^{3}$

(Received 20 January 2009; revised 24 January 2010)

\begin{abstract}
The time varying flow in which fluid is withdrawn from a reservoir through a point sink of variable strength beneath a free surface is considered. Asymptotic techniques are used to derive an approximate solution to the flow that is valid at intermediate times, giving a simple rational approximation to track changes in the free surface for any temporal variations in the sink strength. Comparisons with numerical simulations are given, showing that the approximation has wide applicability.
\end{abstract}

\section{Contents}

1 Introduction 1446-8735. (Print two pages per sheet of paper.) 
3 Weakly non-linear solution

3.1 Leading order . . . . . . . . . . . . . . . . . E22

3.2 Second order solutions . . . . . . . . . . . . E E25

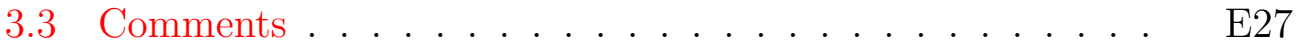

4 Rational approximation $\quad$ E28

5 Numerical solution $\quad$ E29

5.1 Results . . . . . . . . . . . . . . . E E30

6 Conclusions

E32

References

E33

\section{Introduction}

There has been a large amount written about the process of selective withdrawal from stratified reservoirs. In this process, water draws from the main water body in a layer: less dense water (warmer, fresh) above the layer is too buoyant to reach the outlet; and more dense water (colder, salty) below the layer is too heavy to be pulled up. The thickness of the layer depends on the strength of the flow rate and the density gradient. Stronger flow results in a thicker layer, while a stronger density gradient leads to a thinner layer. The general rules of this if the stratification is continuous and reasonably linear have been determined through theoretical, numerical, experimental and field work and are summarized in a number of articles [3, 17, 27, 29].

If the water body consists of layers of different density separated by a thin interface, then a similar process occurs, but the bounds of the withdrawal zone coincide with the interfaces between the density layers in the fluid, and rather than being a continuous variation in withdrawal layer thickness there are jumps at discrete values of flow rate. Beneath a critical flow rate, the withdrawn water will all come from the layer adjacent to the outlet, 
whereas above this rate some may come from other layers as well. This critical transition (often called 'critical drawdown') is important in water quality management of reservoirs and cooling ponds, as there are many situations in which it is desirable to keep the flow rate below the critical value in order to withdraw a layer of pollutant or extract salt water without removing potable supplies (or vice versa). There is again a considerable literature on this problem, both from a theoretical and an experimental standpoint $[2,11,12$, 20, 24].

Early theoretical work consisted mainly of studies of steady state solutions, since these are relatively easier to compute. Slow flow solutions that contain a stagnation point on the surface above the sink exist up to some limiting value that often depends on the geometry. However, this limiting value does not always coincide with the critical drawdown flow rate as described above, and solutions with a downward cusp appear to exist at a somewhat higher value. Cusped solutions were originally thought to be the critical drawdown flows, and strong evidence for this was given by Forbes et al. [7, 14, 15]. In spite of these results, uncertainty remains about the critical flows due to poor comparison with experiment and the existence of multiple cusped solutions for certain geometries, as seen in the work of Vanden-Broeck and Keller [26] and Hocking [12, 13]. Furthermore, what happens in the range of flow rate between the limiting stagnation point flows and the cusped flows is still not clear.

In order to investigate this further, work progressed to consider the unsteady flow situation, allowing the flow to evolve from rest [21, 22, 23, 25, 28, 30]. The effects of neglecting viscosity and a thick interface were considered by Farrow and Hocking [4]. In all of this work the critical drawdown rates appeared to depend on the history of the flow. For example, drawdown of the surface could be initiated by turning on the outlet to a high flow rate very rapidly, leading to a dip in the surface that immediately entered the outlet. On the other hand, Stokes et al. [23] showed that a slow, ramped increase in outflow could often delay the drawdown to higher flow rates than that at which the rapid drawdown occurred. 
The study of withdrawal through a more localized outlet such as a point sink followed a similar historical path. Steady solutions containing a stagnation point directly above the sink were found up to some limiting value. In fluids of both unbounded and finite depth, Forbes et al. [6] and Hocking et al. [16] showed that these solutions reached a limiting form in which a secondary stagnation ring formed on the surface a short distance from the primary, central stagnation point. However, no solutions containing the three dimensional equivalent of a cusp have ever been obtained in the axisymmetric cases considered, in spite of considerable effort (Forbes and Hocking [8]). Simulations of unsteady flows that are capable of accurately examining the flow close to the limiting cases have only recently become available through the work of Miloh and Tyvand [19] and Stokes et al. [22], for example.

We consider the evolution of the interface when the withdrawal rate varies but remains beneath the critical drawdown value. This reflects the true situation in reservoirs or cooling ponds, in which the withdrawal flow is almost never steady for very long and varies through the day as the demands on the system change [17]. A linearised solution is derived and used to develop a simple rational approximation to the evolution of the interface. A range of flow scenarios are considered in the following sections and compared with full simulations, with very encouraging results.

\section{Problem formulation}

We consider the unsteady, irrotational and axisymmetric flow of an inviscid, incompressible fluid of infinite depth into a point sink located beneath a free surface. The sink is initially a depth $h$ in dimensional units beneath the undisturbed level of the free surface and has strength $m(\tau)$, a function of time $\tau$. This problem was considered numerically by Stokes et al. [22], but only in the context of determining critical Froude numbers to guarantee a drawdown of the surface. Here our interests are quite different: we want to know the behaviour of the surface at low flow rates when subject to small 
variations in sink strength, such as might be experienced in a real water storage reservoir. For example, if the sink strength varies slowly, does the free surface move through a sequence of steady states, as conjectured in earlier work by Stokes et al. [21, 22]?

The consequences of the above assumptions are that we can define a velocity potential $\Phi(\hat{r}, \hat{z})$ in polar coordinates, such that $\hat{\mathbf{v}}=\nabla \Phi$ is the velocity vector for the flow. The potential satisfies

$$
\nabla^{2} \Phi=0, \quad \hat{z}<\mathrm{N}(\hat{\mathrm{r}}, \tau)
$$

throughout the fluid domain except at the point $(\hat{r}, \hat{z})=(0,-h)$, the location of the singularity representing the point sink, and where $\hat{z}=N(\hat{r}, \tau)$ is the equation of the free surface. In order to introduce the point sink, the velocity potential must have the limiting behaviour

$$
\Phi \rightarrow \frac{m}{4 \pi} \frac{1}{\sqrt{\hat{r}^{2}+(\hat{z}+h)^{2}}} \quad \text { as } \quad(\hat{r}, \hat{z}) \rightarrow(0,-h) .
$$

The conditions on the free surface are given by the dynamic condition of atmospheric pressure on the free surface, which comes from the Bernoulli equation,

$$
\Phi_{\tau}+\frac{1}{2}\left(\hat{u}^{2}+\hat{v}^{2}\right)+g N=0 \quad \text { on } \hat{z}=N(\hat{r}, \tau),
$$

and the kinematic condition,

$$
\mathrm{N}_{\tau}+\Phi_{\widehat{r}} \mathrm{~N}_{\widehat{\mathrm{r}}}-\Phi_{\hat{z}}=0 \quad \text { on } \hat{z}=\mathrm{N}(\hat{\mathrm{r}}, \tau) .
$$

Note that in a reservoir setting, the free surface is more likely to be an interface between two fluid layers of different density. However, if the upper fluid is assumed to be stagnant and the interface to be sharp, then Yih [29] noted that the equations are the same except that gravity $\mathrm{g}$ can be replaced by the reduced gravity $g^{\prime}=\Delta \rho g / \rho_{0}$, where $\Delta \rho$ is the density difference between the layers and $\rho_{0}$ is some reference density. 
We are interested in the effect of allowing the sink strength $m$ to vary, so we do not include this in our non-dimensionalisation. Instead, we choose a length scale $h$, a time scale $\sqrt{h / g}$, a velocity scale of $\sqrt{g h}$, and a velocity potential scale of $h \sqrt{g h}$. (A different approach was taken for the two dimensional finite depth problem by Stokes et al. [23], leading to a rather different formulation: although equivalent, the approach here is simpler.)

The dimensionless equations are then

$$
\nabla^{2} \phi=0, \quad z<\eta(r, t), \quad(r, z) \neq(0,-1),
$$

subject to

$$
\phi_{\mathrm{t}}+\frac{1}{2}\left(\mathrm{u}^{2}+v^{2}\right)+\eta=0 \quad \text { on } z=\eta(\mathrm{r}, \mathrm{t})
$$

and

$$
\eta_{\mathrm{t}}+\phi_{\mathrm{r}} \eta_{\mathrm{r}}-\phi_{z}=0 \quad \text { on } \mathrm{y}=\eta(\mathrm{r}, \mathrm{t})
$$

with the extra condition that

$$
\phi \rightarrow \frac{\mathrm{F}(\mathrm{t})}{\sqrt{\mathrm{r}^{2}+(z+1)^{2}}} \quad \text { as }(\mathrm{r}, z) \rightarrow(0,-1), \quad \mathrm{t}>0,
$$

where the Froude number is defined to be the function of time

$$
F(t)=\frac{m(t)}{4 \pi \sqrt{g h^{5}}} .
$$

The only important quantity is then the Froude number $F(t)$, whose variation is caused only by changes in the sink strength, $m$.

\section{$3 \quad$ Weakly non-linear solution}

In this section, we assume a small displacement of the interface or free surface, and find an approximate solution consisting of successive linearizations. Consider the equations $(5,6,7)$ with (8). Linearizing about $z=0$, that is, assuming both the Froude number and the surface disturbance are small, we get the 
following cascade of linear problems. Assuming the Froude number is given by $F(t)=\epsilon F_{1}(t)$ and letting $\phi=\epsilon \phi_{1}+\epsilon^{2} \phi_{2}+\cdots$ and $\eta=\epsilon \eta_{1}+\epsilon^{2} \eta_{2}+\cdots$, gives

$$
\begin{aligned}
\mathcal{O}(\epsilon): & \nabla^{2} \phi_{1}=0, \quad-\infty<z<0, \quad 0 \leqslant \mathrm{r}<\infty, \quad \mathrm{t}>0, \\
& \eta_{1 \mathrm{t}}-\phi_{1 z}=0 \quad \text { on } z=0, \\
& \phi_{1 \mathrm{t}}+\eta_{1}=0 \quad \text { on } z=0, \\
& \phi_{1}(\mathrm{r}, z, \mathrm{t}) \rightarrow \frac{\mathrm{F}_{1}(\mathrm{t})}{\left(\mathrm{r}^{2}+(z+1)^{2}\right)^{1 / 2}} \quad \text { as } z \rightarrow-1 ; \\
\mathcal{O}\left(\epsilon^{2}\right): \quad & \nabla^{2} \phi_{2}=0, \quad-\infty<z<0, \quad 0 \leqslant \mathrm{r}<\infty, \quad \mathrm{t}>0, \\
& \eta_{2 \mathrm{t}}-\phi_{2 z}=-\phi_{1 \mathrm{r}} \eta_{1 \mathrm{r}} \quad \text { on } z=0, \\
& \phi_{2 \mathrm{t}}+\eta_{2}=-\frac{1}{2}\left(\phi_{1 \mathrm{r}}^{2}+\phi_{1 z}^{2}\right) \quad \text { on } z=0 .
\end{aligned}
$$

\subsection{Leading order}

To solve the leading order problem, we choose a particular form for the solution. Equations $(10,11)$ transform to

$$
\phi_{1 \mathrm{tt}}+\phi_{1 z}=0 \quad \text { on } \quad z=0,
$$

as in linear water wave theory (Lamb [18, p.364]), and so we choose

$$
\phi_{1}(r, z, t)=\frac{F_{1}(t)}{\left(r^{2}+(z+1)^{2}\right)^{1 / 2}}+\frac{F_{1}(t)}{\left(r^{2}+(z-1)^{2}\right)^{1 / 2}}+\int_{0}^{\infty} A(k, t) e^{k z} J_{0}(k r) d k
$$

which (by separation of variables) satisfies Laplace's equation in cylindrical polar coordinates everywhere below $z=0$, except as $(r, z) \rightarrow(0,-1)$. The first two terms of this expression (involving $F(t)$ ) represent the sink and an image above the free surface. These two terms were shown by Forbes et al. [9] to become the dominant components for flows in which the Froude number was increased linearly to a final value and then maintained at that value thereafter. 
Substituting (17) into the condition (16), gives

$$
\begin{aligned}
& \frac{F_{1}^{\prime \prime}(t)}{\left(r^{2}+(z+1)^{2}\right)^{1 / 2}}+\frac{F_{1}^{\prime \prime}(t)}{\left(r^{2}+(z-1)^{2}\right)^{1 / 2}}+\int_{0}^{\infty} A_{t t}(k, t) e^{k z} J_{0}(k r) d k \\
& +F_{1}(t)\left[\frac{-(z+1)}{\left(r^{2}+(z+1)^{2}\right)^{3 / 2}}-\frac{(z-1)}{\left(r^{2}+(z-1)^{2}\right)^{3 / 2}}\right] \\
& +\int_{0}^{\infty} k A(k, t) e^{k z} J_{0}(k r) d k=0
\end{aligned}
$$

and therefore on $z=0$ we have

$$
\frac{2 F_{1}^{\prime \prime}(t)}{\left(r^{2}+1\right)^{1 / 2}}+\int_{0}^{\infty}\left(A_{t t}+k A\right) J_{0}(k r) d k=0 .
$$

Using the integral (Gradshteyn and Ryshik [10])

$$
\int_{0}^{\infty} e^{-k} J_{0}(r k) d k=\left(r^{2}+1\right)^{-1 / 2}
$$

we find

$$
\int_{0}^{\infty} G(k, t) J_{0}(k r) d k=-\frac{2 F_{1}^{\prime \prime}(t)}{\left(r^{2}+1\right)^{1 / 2}}
$$

and so

$$
\mathrm{G}(\mathrm{k}, \mathrm{t})=-2 \mathrm{~F}_{1}^{\prime \prime}(\mathrm{t}) e^{-\mathrm{k}} .
$$

Therefore, $A(k, t)$ satisfies

$$
A_{\mathrm{tt}}+k A=-2 \mathrm{~F}_{1}^{\prime \prime}(\mathrm{t}) e^{-k},
$$

which depends on the form of $F_{1}(t)$. The solution to this order is therefore

$$
\begin{aligned}
\phi_{1}(r, z, t)= & \frac{F_{1}(t)}{\left(r^{2}+(z+1)^{2}\right)^{1 / 2}}+\frac{F_{1}(t)}{\left(r^{2}+(z-1)^{2}\right)^{1 / 2}} \\
& +\int_{0}^{\infty} A^{*}(k, t) e^{k z} J_{0}(k r) d k
\end{aligned}
$$




$$
+\int_{0}^{\infty}\left[c_{0}(k) \cos \sqrt{k} t+d_{0}(k) \sin \sqrt{k} t\right] e^{k z} J_{0}(k r) d k
$$

where $A^{*}(k, t)$ is the particular solution to (23) and the last term represents the terms due to the initial conditions on the surface.

Equation (11) evaluated on $z=0$ provides the leading order expression for the evolution of the free surface as

$$
\begin{aligned}
\eta_{1}(r, t)= & \frac{-2 F_{1}^{\prime}(t)}{\left(r^{2}+1\right)^{1 / 2}}-\int_{0}^{\infty} A_{t}^{*}(k, t) J_{0}(k r) d k \\
& +\int_{0}^{\infty}\left[c_{0}(k) \sin \sqrt{k} t-d_{0}(k) \cos \sqrt{k} t\right] J_{0}(k r) k^{1 / 2} d k
\end{aligned}
$$

The forms of $c_{0}(k)$ and $d_{0}(k)$ are determined by the initial conditions on $z=0$ for both $\phi_{1}$ and $\eta_{1}$. For example, the standard starting conditions $\phi_{1}(r, 0,0)=0$ and $\eta_{1}(r, 0)=0$ give

$$
\begin{aligned}
& \phi_{1}(r, 0,0)=0=\frac{2 F_{1}(0)}{\left(r^{2}+1\right)^{1 / 2}}+\int_{0}^{\infty} A^{*}(k, 0) J_{0}(k r) d k+\int_{0}^{\infty} c_{0}(k) J_{0}(k r) d k \\
& \eta_{1}(r, 0)=0=\frac{-2 F_{1}^{\prime}(0)}{\left(r^{2}+1\right)^{1 / 2}}-\int_{0}^{\infty} A_{t}^{*}(k, 0) J_{0}(k r) d k-\int_{0}^{\infty} d_{0}(k) J_{0}(k r) d k,
\end{aligned}
$$

giving $c_{0}(k)=-2 F(0) e^{-k}$ and $d_{0}(k)=-2 F_{1}(0) e^{-k} k^{-1 / 2}$. The method of stationary phase shows that these terms decay to zero as $t \rightarrow \infty$, and integration by parts shows that this occurs like $\mathcal{O}\left(t^{-2}\right)$. Thus these terms are transient (and decay quite quickly).

The particular solution for (23) can easily be shown to be

$$
A^{*}(k, t)=\frac{2 e^{-k}}{\sqrt{k}} \int_{0}^{t} F_{1}^{\prime \prime}(\xi) \sin [\sqrt{k}(\xi-t)] d \xi
$$

which when substituted into (24) gives the shape of the interface to this order. In general, this calculation becomes intractable. 
However, we assume that the sink strength function is $F(t)=\epsilon F_{1}(t)=\beta t=$ $\epsilon \beta_{1} t$, so that the increase in withdrawal strength is linear with time, so that $\phi_{1}(r, z, t)=\frac{2 \beta_{1} t}{\left(r^{2}+1\right)^{1 / 2}}+\int_{0}^{\infty}\left[c_{0}(k) \cos \sqrt{k} t+d_{0}(k) \sin \sqrt{k} t\right] e^{k z} J_{0}(k r) d k$

and

$$
\eta_{1}(r, t)=\frac{-2 \beta_{1}}{\left(r^{2}+1\right)^{1 / 2}}+\int_{0}^{\infty}\left[c_{0}(k) \sin \sqrt{k} t-d_{0}(k) \cos \sqrt{k} t\right] J_{0}(k r) k^{1 / 2} d k
$$

since $F_{1}^{\prime \prime}(t)=0$ and therefore $A^{*}(k, t)$ is identically zero. Note that this provides what amounts to a 'steady' solution, in the sense that if the transient component due to the initial conditions dies out quickly the first order solution gives a surface that maintains a constant dip. Simulations (see later) demonstrate the surface remains apparently motionless for some time even as the flow rate increases.

\subsection{Second order solutions}

Proceeding as before to the second order, the velocity potential takes the form

$$
\phi_{2}(r, z, t)=\int_{0}^{\infty} A_{1}(k, t) e^{k z} J_{0}(k r) d k
$$

which satisfies Laplace's equation. On substitution of the known leading order terms into equations $(14,15)$, we find

$$
\phi_{2 t \mathrm{t}}+\phi_{2 z}=-8 \frac{\mathrm{F}_{1}(\mathrm{t}) \mathrm{F}_{1}^{\prime}(\mathrm{t}) \mathrm{r}^{2}}{\left(\mathrm{r}^{2}+1\right)^{3}}
$$

Again substituting $z=0$ gives

$$
\int_{0}^{\infty}\left(A_{1 t t}+k A_{1}\right) J_{0}(k r) d k=-8 \frac{F_{1}(t) F_{1}^{\prime}(t) r^{2}}{\left(r^{2}+1\right)^{3}}
$$


The solution to this is $A_{1}(k, t)=A_{1 C F}(k, t)+A_{1}^{*}(k, t)$ where $A_{1}^{*}(k, t)$ is the particular solution to $A_{1 \mathrm{tt}}+k A_{1}=g_{1}(k, t)$ where $g_{1}(k, t)$ satisfies

$$
\int_{0}^{\infty} g_{1}(k, t) J_{0}(k r) d k=-8 \frac{F_{1}(t) F_{1}^{\prime}(t) r^{2}}{\left(r^{2}+1\right)^{3}}
$$

After some work, one obtains

$$
g_{1}(k, t)=-8 F_{1}(t) F_{1}^{\prime}(t) k^{1 / 2} \mathcal{H}\left\{\frac{r^{3 / 2}}{\left(r^{2}+1\right)^{3}}\right\}
$$

where $\mathcal{H}\{\}$ denotes the Hankel transform [10].

Continuing we obtain

$$
k^{-1 / 2} g_{1}(k, t)=-F_{1}(t) F_{1}^{\prime}(t) k^{5 / 2} K_{0}(k)
$$

where $K_{0}(k)$ is the modified Bessel function [1], and consequently, $A_{1}^{*}$ satisfies

$$
A_{1 \mathrm{tt}}+\mathrm{k} A_{1}=-\mathrm{F}_{1} \mathrm{~F}_{1}^{\prime} \mathrm{k}^{3} \mathrm{~K}_{0}(\mathrm{k}) .
$$

Again, taking the case of a linear ramping of withdrawal rate, $F(t)=\epsilon F_{1}(t)=$ $\beta t=\epsilon \beta_{1} t$, to get the solution $A_{1}^{*}=-\beta_{1}^{2} k^{2} K_{0}(k) t$, and assuming that nontrivial initial conditions are of leading order only we find

$$
\phi_{2}(r, z, t)=-\beta_{1}^{2} t \int_{0}^{\infty} k^{2} e^{k z} J_{0}(k r) d k
$$

and therefore,

$$
\phi_{2}(r, 0, t)=-2 \beta_{1}^{2} \operatorname{tr}\left(r^{2}+1\right)^{-2} .
$$

Substituting back into the second order surface condition then gives

$$
\eta_{2}(r, t)=-\phi_{2 t}-\frac{1}{2} \phi_{1 r}^{2}=\frac{2 \beta_{1}^{2}}{\left(r^{2}+1\right)^{3}}\left(r^{3}+r-r^{2} t^{2}\right) .
$$

Thus, the solution for $\eta(r, t)$ to second order is

$$
\eta(r, t)=\epsilon \eta_{1}(r, t)+\epsilon^{2} \eta_{2}(r, t)=\frac{-2 \epsilon \beta_{1}}{\left(r^{2}+1\right)^{1 / 2}}+\frac{2 \epsilon^{2} \beta_{1}^{2}}{\left(r^{2}+1\right)^{3}}\left(r^{3}+r-r^{2} t^{2}\right)
$$


and so we have

$$
\eta(r, t)=\frac{-2 \beta}{\left(r^{2}+1\right)^{1 / 2}}+\frac{2 \beta^{2}}{\left(r^{2}+1\right)^{3}}\left(r^{3}+r-r^{2} t^{2}\right) .
$$

This last equation is usefully expressed in terms of $F(t)$ as

$$
\eta(r, t)=\frac{-2 F^{\prime}(t)}{\left(r^{2}+1\right)^{1 / 2}}-\frac{2 r^{2} F(t)^{2}}{\left(r^{2}+1\right)^{3}}+\frac{2 F^{\prime}(t)^{2}\left(r^{3}+r\right)}{\left(r^{2}+1\right)^{3}}
$$

\subsection{Comments}

The solution (34) represents a very simple form for the evolution of the free surface provided the form of $F(t)$ is linear and the surface displacement is small. As time increases the displacement increases and the solution loses its validity. However, agreement with simulations is very good.

This solution holds for the case of a linear increase in the sink strength. It shows a significant component that is steady if there is a linear ramping of the inflow rate, as it involves the first derivative of the time dependent Froude number. A dip forms that is proportional in size to the rate of change of $F(t)$ with time, corresponding to the first term in both (34) and (35). The final term in (35) likewise represents a constant term, albeit one of much smaller magnitude than the first.

Of course this solution only has validity for the case in which $F(t)=\beta t$ for small $\beta$, and then only for relatively small time. The second term becomes of similar order to the first when $t \sim \mathcal{O}\left(\beta^{-1 / 2}\right)$, suggesting an upper bound on the temporal validity. However, note that the middle term in (35) is the asymptotic (to second order in F) stagnation point steady state surface that would evolve in response to a constant value of $F[5]$; this suggests that providing $F(t)$ is not too large, a component having the form of a stagnation point steady flow will play a role in the free surface profile. 


\section{Rational approximation}

Our goal in this work is to obtain a rational function that will provide a reasonable approximation to the behaviour of the surface as the inflow rate varies in some general manner. The solution obtained above only applies for a linear increase (or decrease) in Froude number, or flow rate. However, if the second derivative of the small Froude number $F(t)$ is always small in comparison to $F(t)$, then locally, $F(t)$ looks like a linear ramp-up (or down), and the approximation continues to work well.

Approximating $F(t)$ by a piecewise linear function, if the slopes of the successive pieces are close enough to one-another, then the 'local transient phases' will be extremely short lived. Informally taking the limit as the intervals of constant slope approach zero in length, the equations for the surface shape are modified by replacing $\beta t$ by the more general form $F(t)$. Using equation (6) to leading order for general $F(t)$, we obtain

$$
\begin{aligned}
\eta & =-\phi_{t}-\frac{1}{2}\left(u^{2}+v^{2}\right) \\
& \approx-F^{\prime}(t) \phi_{0}(r, 0)-\frac{1}{2} F(t)^{2} \phi_{0 r}(r, 0)^{2} \\
& =-F^{\prime}(t) y_{1}(r)-F(t)^{2} y_{2}(r),
\end{aligned}
$$

where

$$
y_{1}(r)=\phi_{0}(r, 0)=\frac{2}{\left(r^{2}+1\right)^{1 / 2}}
$$

and

$$
y_{2}(r)=\phi_{o r}(r, 0)^{2} / 2=\frac{2 r^{2}}{\left(r^{2}+1\right)^{3}}
$$

The term $-F^{\prime}(t) y_{1}(r)$ is a dip (or bulge if $F^{\prime}(t)<0$ ) centred on $r=0$ and proportional in size to the rate of change of $F(t)$, whereas the term $-F(t)^{2} y_{2}(r)$ is the familiar asymptotic (to second order in $\mathrm{F}$ ) stagnation point steady state 
surface that would evolve at the current value of F. So both terms have some physical interpretation in the context of the problem.

This form omits the terms of order $F^{\prime \prime}(t)$ that arise in the above calculations but provided these are small then we might expect the solution to be a reasonable approximation to the unsteady behaviour for a general $F(t)$. For the case in which $F(t)=\beta t$, discussed previously, only the negligible non-time dependent order $F^{\prime}(t)^{2}$ term appearing in Equation (35) is absent, suggesting that the current rational approximation can indeed be viewed as a piecewise linear application of the results derived for the linear ramp-up.

In what follows, we compare this rational approximation with a full simulation of the evolution of the free surface.

\section{$5 \quad$ Numerical solution}

A method for the numerical solution of the time dependent problem was described in the recent article by Stokes et al. [22]. However, for completeness, we briefly summarize the method. The full equations are standard for unsteady free-surface hydrodynamics, that is (5), (6), (7) with the addition of the singularity representing the point sink flow, (8).

This system is solved by placing ring source/sink singularities (that satisfy Laplace's equation within the fluid) just above a discrete representation of the interface at $z_{j}=\eta_{j}\left(r_{j}\right)+\delta_{j}, j=1,2, \ldots, N$, where $\delta_{j}, j=1,2,3, \ldots$, are determined to provide optimal performance [22]. The strengths of these source/sink singularities are allowed to evolve in time in order to satisfy the flow conditions. In what follows, initial conditions are required to get started, and the choice made here is

$$
\phi(r, z, 0)=0 \text { and } \eta(r, 0)=0
$$

in keeping with other work on this problem [9, e.g.]. This represents a flow initiated from a quiescent situation. 


\section{$5.1 \quad$ Results}

Full simulations of the flow in which the sink is turned on and varied in strength according to various forms of $F(t)$ are generated, and compared with the rational approximation given above. The results of linear ramping, an exponential levelling off, a linear ramping followed by a 'jump' to a cubic increase, and sinusoidal oscillations in flow rate are shown in accompanying animations (see supplementary files at the web site). In each case the blue line is the full simulation and the red line is the rational approximation for the same flow.

In the first animation ${ }^{1}$ the Froude number

$$
F(t)=t / 200
$$

is increased linearly from zero. The rational approximation (red) appears to be almost in a steady state configuration while the transients of the full simulation (blue) evolve until the two interfaces match, after which both begin to travel downward as the full simulation approaches drawdown (the rational approximation cannot give this outcome). In the full simulation the transient phase dies out very quickly, after which the rational approximation is excellent.

The second animation ${ }^{2}$ shows the situation

$$
\mathrm{F}(\mathrm{t})=0.02[1-\exp (-\mathrm{t} / 25)]
$$

Again the solutions match exceedingly well once the initial transient phase has passed. The dip forms as above, and as suggested by the asymptotic approach, it is of the size predicted by the first derivative of $F(t)$. Therefore,

${ }^{1}$ http://anziamj .austms.org.au/ojs/index.php/ANZIAMJ/article/ downloadSuppFile/1717/7562; this and the other animations usually can be viewed within a web browser.

${ }^{2}$ http://anziamj . austms.org.au/ojs/index.php/ANZIAMJ/article/ downloadSuppFile/1717/7564 
as the sink strength levels off, the size of the dip decreases. This exemplifies again how treating the simulation as a sequence of linear segments is reasonable. After this initial phase, the quadratic term begins to play a role and as the value of $F(t) \rightarrow 0.02$ as $t \rightarrow \infty$, both the simulation and the rational approximation approach the steady state solution (shown in green) for the value $F=0.02$ [5]. The value of Froude number in this simulation remains well below the critical drawdown value for all time.

The third animation ${ }^{3}$ reveals the behaviour when there is a change in the form of $F(t)$. In this third example, the linear ramp-up as discussed earlier is applied from $t=0$ to $t=20$, at which point $F=0.02$. After that, $a$ cubic function is added on, shifted to start at $t=20$ so that $F(t)$ is twice differentiable for all $t>0$. Thus

$$
F(t)=t / 1000+H_{20}(t)(t-20)^{3} / 400000
$$

where $H_{a}(t)$ is the Heaviside step function which is 0 for $t \leqslant a$ and 1 for $\mathbf{t}>\mathbf{a}$. The steady component of the rational approximation is quickly joined by the numerical solution during the linear phase, and then, as the influence of the cubic component becomes apparent, the surface dips downward. The important point here is that there is no apparent transient phase in moving from the linear to the cubic ramp-up (as there is no 'start-up' from quiescence to pass through), and moreover the match between the numerics and our approximation is excellent for much of the cubic phase as well, highlighting the relevance of the approximation providing $F^{\prime \prime}(t)$ is small.

Finally, we consider a case for which the rational approximation might be expected to be less applicable, given the form of $F(t)$. The choice of flow rate for this animation ${ }^{4}$ was

$$
F(t)=\frac{1}{20}\left[1-\cos \left(\frac{2 \pi t}{50}\right)\right] .
$$

\footnotetext{
${ }^{3}$ http://anziamj . austms . org.au/ojs/index.php/ANZIAMJ/article/ downloadSuppFile/1717/7565

${ }^{4}$ http://anziamj . austms.org.au/ojs/index.php/ANZIAMJ/article/ downloadSuppFile/1717/7566
} 
Sometimes the sink is actually pumping outward, that is behaves like a source (all approximations are still valid for negative sink strength values), and yet again the rational approximation is surprisingly good. The phase is exactly correct and the amplitude is slightly underestimated.

In all of the animations the rational approximation does a very good job of resolving the shape of the free surface once the initial transients due to turning on the sink have passed, providing $F^{\prime \prime}(t)$ remains reasonably small. In almost all reservoir situations, this is a valid assumption.

\section{Conclusions}

A rational approximation is provided via a perturbation expansion to the problem of a point sink in a fluid of infinite depth that is varying in strength. The process described in this article will not work in two dimensions with a line sink in a fluid of infinite depth because of the logarithmic nature of the potential function: the same analysis can be attempted but does not give physically meaningful results (one obtains a logarithmic $\eta_{1}$ term leading to an unbounded elevation in the far field).

In three dimensions, however, the rational function approximation compares very well with the full simulation history of the flow at intermediate times, even if the flow is changed quite quickly. The apparent region of validity is much greater than that expected from the restrictions on the calculations, and provides a reasonable representation of the flow variations except when the critical drawdown point is reached. However, this is a highly non-linear process and one would not expect the linear theory to predict this event. Bearing in mind that even the full simulations are an approximation to real flows, the results suggest that in the 'field' case, where the flow rate is varying constantly, the rational function model is a very good indicator of the behaviour of the interface of such a two layer fluid. This is expected to be of use in the practical management and monitoring of reservoir operation. 


\section{References}

[1] M. Abramovitz and I. Stegun, Handbook of Mathematical Functions, Dover, New York, 1972 E26

[2] A. Craya, 1949, Theoretical research on the flow of nonhomogeneous fluids, La Houille Blanche, 4, 44-55. E18

[3] W. R. Debler, 1959, Stratified flow into a line sink, J. Eng. Mech. Div., $A S C E$ 3, 51-65 E17

[4] D. E. Farrow and G. C. Hocking, 2006, A numerical model for withdrawal from a two layer fluid, J. Fluid Mech. 549, 141-157. doi:10.1017/S0022112005007561 E18

[5] L. K. Forbes and G. C. Hocking, 1990, Flow caused by a point sink in a fluid having a free surface, J. Austral. Math. Soc. Ser. B 32, 233-252. doi:10.1017/S0334270000008465 E27, E31

[6] L. K. Forbes, G. C. Hocking and G. A. Chandler, 1996, A note on withdrawal through a point sink in fluid of finite depth, J. Austral. Math. Soc. Ser. B 37, 406-416. doi:10.1017/S0334270000008961 E19

[7] L. K. Forbes and G. C. Hocking, 1998, Withdrawal from a two-layer inviscid fluid in a duct, J. Fluid Mech. 361, 275-296. doi:10.1017/S0334270000010742 E18

[8] L. K. Forbes and G. C. Hocking, 2003, On the computation of steady axi-symmetric withdrawal from a two-layer fluid, Computers and Fluids, 32, 385-401. doi:10.1017/S0022112098008805 E19

[9] L. K. Forbes, G. C. Hocking and T. E. Stokes, 2008, On starting conditions for a submerged sink in a fluid, J. Eng. Math., 61, 55-68. doi:10.1007/s10665-007-9174-2 E22, E29

[10] I. S. Gradshteyn and I. W. Ryzhik, Table of integrals series and products, Academic Press, New York \& London, 1965. E23, E26 
[11] G. Hocking, 1985, Cusp-like free-surface flows due to a submerged source or sink in the presence of a flat or sloping bottom, J. Aust. Math Soc. Ser. B, 26, 470-486. doi:10.1017/S0334270000004665 E18

[12] G. Hocking, 1991, Withdrawal from two-layer fluid through line sink, J. Hyd. Engng., ASCE, 117(6), 800-805. doi:10.1061/(ASCE)0733-9429(1991)117:6(800) E18

[13] G. Hocking, 1991, Critical withdrawal from a two-layer fluid through a line sink, J. Eng. Math., 25, 1-11. doi:10.1007/BF00036598 E18

[14] G. Hocking, 1995, Supercritical withdrawal from a two-layer fluid through a line sink, J. Fluid Mech., 297, 37-47. doi:10.1017/S022112095002990 E18

[15] G. C. Hocking and L. K. Forbes, 2001, Supercritical withdrawal from a two-layer fluid through a line sink if the lower layer is of finite depth, J. Fluid Mech. 428, 333-348. doi:10.1017/S0022112000002780 E18

[16] G. C. Hocking, J-M. Vanden Broeck and L. K. Forbes, 2002, Withdrawal from a fluid of finite depth through a point sink, ANZIAM J., 44, 181-191. doi:10.1017/S1446181100013882 E19

[17] J. Imberger and J. C. Patterson, 1990, Physical Limnology, Adv. in Appl. Mech., 27, 303-475 E17, E19

[18] H. Lamb, Hydrodynamics, Cambridge Univ. Press, New York, 6th ed. 1993 E22

[19] T. Miloh and P. A. Tyvand, 1993, Nonlinear transient free-surface flow and dip formation due to a point sink, Phys. Fluids A 5 (6), 1368-1375. doi:10.1063/1.858572 E19

[20] C. Sautreaux, 1901, Mouvement d'un liquide parfait soumis à lapesanteur. Dé termination des lignes de courant, J. Math. Pures Appl. 7 (5), 125-159. E18 
[21] T. E. Stokes, G. C. Hocking and L. K. Forbes, 2003, Unsteady free-surface flow induced by a line sink, J. Eng. Maths, 47, 137-160. doi:10.1023/A:1025892915279 E18, E20

[22] T. E. Stokes, G. C. Hocking and L. K. Forbes, 2005, Unsteady flow induced by a withdrawal point beneath a free surface, ANZIAM J., 47, 185-202. doi:10.1017/S1446181100009986 E18, E19, E20, E29

[23] T. E. Stokes, G. C. Hocking and L. K. Forbes, 2008, Unsteady free surface flow induced by a line sink in a fluid of finite depth, Computers and Fluids, 37, 236-249. doi:10.1016/j.compfluid.2007.06.002 E18, E21

[24] E. O. Tuck, and J-M. Vanden Broeck, 1984, A cusp-like free-surface flow due to a submerged source or sink, J. Aust. Math Soc. Ser. B, 25, 443-450. doi:10.1017/S0334270000004197 E18

[25] P. A. Tyvand, 1992, Unsteady free-surface flow due to a line source, Phys. Fluids A, 4 (4), 671-676. doi:10.1063/1.858285 E18

[26] J-M. Vanden Broeck and J. B. Keller, 1987, Free surface flow due to a sink, J. Fluid Mech., 175, 109-117. doi:10.1017/S022112087000314 E18

[27] I. R. Wood, 1968, Selective withdrawal from a stably stratified fluid, J. Fluid Mech., 32, 209-223. doi:10.1017/S022112068000686 E17

[28] M. Xue and D. K. P. Yue, 1998, Nonlinear free-surface flow due to an impulsively started submerged point sink, J. Fluid Mech., 364, 325-347. doi:10.1017/S022112098001335 E18

[29] C. S. Yih, Dynamics of nonhomogeneous fluids, Macmillan, New York, 1965. E17, E20

[30] Q-N. Zhou and W. P. Graebel, 1990, Axisymmetric draining of a cylindrical tank with a free surface, J. Fluid. Mech. 221, 511-532. doi:10.1017/S022112090003652 E18 


\section{Author addresses}

1. G. C. Hocking, Mathematics and Statistics, Murdoch University, Murdoch, 6150, Western Australia mailto:G.Hocking@murdoch.edu . au

2. T. E. Stokes, University of Waikato mailto:stokes@math.waikato .ac.nz

3. L. K. Forbes, University of Tasmania mailto: larry. forbes@utas .edu.au 